\title{
PRELIMINARY EVALUATION OF GAOFEN-3 POLARIMETRIC AND RADIOMETRIC ACCURACY BY CORNER REFLECTORS IN INNER MONGOLIA
}

\author{
L. Shi ${ }^{1 *}$, X. Ding ${ }^{2}$, P. Li ${ }^{1}$, J. Yang ${ }^{1}$, L. Zhao ${ }^{3}$, L. Yang ${ }^{1}$, Y. Chang ${ }^{1}$, L. Yan ${ }^{4}$ \\ ${ }^{1}$ State Key Laboratory of Information Engineering in Surveying, Mapping and Remote Sensing, Wuhan University, China \\ shi.lei@whu.edu.cn \\ 2 Department of Land Surveying and Geo-Informatics, Hong Kong Polytechnic University, Kowloon, Hong Kong \\ ${ }^{3}$ School of Remote Sensing and Information Engineering, Wuhan University, China \\ ${ }^{4}$ China Centre For Resources Satellite Data and Application
}

Commission ICWG III/IVb

KEY WORDS: Gaofen-3, Quality evaluation, Polarimetric and Radiometric accuracy

\begin{abstract}
:
On August 10, 2016, China launched its first C-band full polarimetric radar satellite, named Gaofen-3 (GF-3), for urban and agriculture monitoring, landslide detection, ocean applications, etc. According to the design specification, GF-3 is expected to work at $-35 \mathrm{~dB}$ crosstalk and $0.5 \mathrm{~dB}$ channel imbalance, with less than 10 degree error. The absolute radiometric bias is expected to be less than $1.5 \mathrm{~dB}$ in a single scene and $2.0 \mathrm{~dB}$ when operating for a long time. To complete the calibration and evaluation, the Institute of Electronics, Chinese Academy Sciences (IECAS) built a test site at Inner Mongolia, and deployed active reflectors (ARs) and trihedral corner reflectors (CRs) to solve and evaluate the hardware distortion. To the best of the authors' knowledge, the product accuracy of GF-3 has not been comprehensively evaluated in any open publication. The remote sensing community urgently requires a detailed report about the product accuracy and stability, before any subsequent application. From June to August of 2017, IECAS begun its second round ground campaign and deployed $10 \mathrm{CRs}$ to evaluate product distortions. In this paper, we exploit Inner Mongolia CRs to investigate polarimetric and radiometric accuracy of QPSI I Stripmap. Although some CRs found fall into AR side lobe, the rest CRs enable us to preliminarily evaluate the accuracy of some special imaging beams. In the experimental part, the image of July 6,2017 was checked by 5 trihedral CRs and the integration estimation method demonstrated the crosstalk varying from -42.65 to $-32.74 \mathrm{~dB}$, and the channel imbalance varying from -0.21 to 0.47 with phase error from -2.4 to 0.2 degree. Comparing with the theoretical radar cross-section of $1.235 \mathrm{~m}$ trihedral CR, i.e. $35 \mathrm{~dB}$, the radiometric error varies about $0.20 \pm 0.29 \mathrm{~dB}$ in $\mathrm{HH}$ channel and $0.40 \pm 0.20 \mathrm{~dB}$ in VV channel.
\end{abstract}

\section{INTRODUCTION}

On August 10, 2016, Chinese new generation C-band highresolution radar satellite, named GaoFen-3 (GF-3), was launched into space. GF-3 carried a new multi-model synthetic aperture radar (SAR) sensor which was designed working at 755 kilometer sun-synchronous orbit (Zhang, 2017), and expected contributing to urban buildings evaluation (Shi, 2015; Sun, 2016), surface deformation $(\mathrm{Hu}, 2017)$ and landslide monitoring, agricultural biomass estimation, ocean wind field estimation (Voronovich, 2017), oil slick detection (Shi, 2016) and ship monitoring, etc. Comparing with current three on-orbit C-band SAR satellites, i.e. RISAT-1, RadarSat-2 and Sentinel-1, GF-3 provides more observation modes.

Before any subsequent GF-3 application, it is critical to assess the radiometric and polarimetric accuracy on public products. Although the GF-3 has been launched into space over one year, there is still absent public evaluation work. Therefore, the assessment program is critical and urgently demand by China remote sensing community. Since sensors launched into space, there were two times ground campaign implemented in Inner Mongolia, north China. The first ground validation begun from September to November of 2016, which mainly tested the image resolution, swath width, peak side lobe ration, radiometric accuracy and geometric accuracy, etc. After the in-orbit hardware inspection and process chain upgrade, the system is believed stable after April of 2017. Then, the second round ground campaign started in June of 2017 to further check the radiometric and polarimetric accuracy.

It should be noticed that there are twelve imaging models designed and more than hundreds of beams are payload on GF-3. The different beams usually present different antenna patterns. It is very time cost to calibrate individual beam in very short satellite passing time. Therefore, the data quality of China Centre For Resources Satellite Data and Application (CCRSDA): http://218.247.138.119:7777/DSSPlatform/productSearch.html is different from beam to beam. Moreover, the calibration campaign is still periodically undergoing, and we also find the inner calibration circuit is not always switched on. To this day, the image quality varies since launched time.

Among all imaging modes, the QPSI I, QPSI II and wave modes could generate full polarimetric observation. The QPSI I mode presents very high resolution, i.e. $8^{*} 6 \sim 9$ meters in range and azimuth direction. In this paper, we collect a GF-3 QPSI I full polarimetric image which was observed on July 6 of 2017 to evaluate the polarimetric and radiometric accuracy.

\section{METHODLOGY}

The size of Inner Mongolia deployed CRs is 1.235 meters which theoretically generate $35 \mathrm{~dB}$ radar cross-section (RCS) under Cband $5.4 \mathrm{GHz}$ carrier wave illuminating. But, the QPSI I image resolution and pixel space is much larger than $1.235 \mathrm{~m}$, and the pixel digital value is contributed by $\mathrm{CR}$ and surrounding 
background clutter. Therefore, this paper utilizes integration estimator (Gray, 1990; Freeman, 1992) to check crosstalk, channel imbalance and RCS levels.

For each CR, we first locate the peak pixel location by the maximum span and select the central $23 \times 23$ image patch for the following process. The small $23 \times 23$ patch is oversampled 16 times to derive the enlarged patch and locate a more precise peak location. A cross with $16 * 16+1$ diameter and $4 * 16+1$ width is subsequently placed on the center of the peak location to ensure all the side lobes of the $\mathrm{CR}$ are involved. If assuming $\sum_{\text {cross }}\left|M_{X Y}\right|^{2}$ is the integrated intensity in the cross window and $N b_{\text {cross }}$ is the number of cross pixels, $\sum_{\text {clutter }}\left|M_{X Y}\right|^{2}$ is the background clutter integrated intensity from the image patch, but not within the cross, and $N b_{\text {clutter }}$ is the corresponding number of pixels. The image-derived $\mathrm{HH}, \mathrm{HV}, \mathrm{VH}$, and VV RCS of the CR are:

$$
\sigma_{X Y}=\left(\sum_{\text {cross }}\left|M_{X Y}\right|^{2}-\frac{N b_{\text {cross }}}{N b_{\text {clutter }}} \sum_{\text {clutter }}\left|M_{X Y}\right|^{2}\right) \frac{P_{r} P_{a}}{\sin \theta} \frac{1}{16^{2}}
$$

$\mathrm{X}$ and $\mathrm{Y}$ is $\mathrm{H}$ or $\mathrm{V}$ channel. If $M_{X Y}$ is stored as the nominal RCS (NRCS) format, i.e., sigma naught, the range, azimuth pixel spaces $P_{r}$ and $P_{a}$, and incidence angle $\theta$ can convert the NRCS to RCS. After implementing the integrated RCS estimator, the GF3-derived $\mathrm{HH}$ and VV channel RCS $\sigma_{h h}$ and $\sigma_{v v}$ can be directly compared with the theoretical value, i.e., about $35 \mathrm{~dB}$, which is from $4 \pi L^{4} / 3 \lambda^{2}$ (Freeman, 1992) by CR size $1.235 \mathrm{~m}$ and radar wavelength $0.056 \mathrm{~m}$. The co-pol channel imbalance intensity and the two crosstalk values of the $\mathrm{CR}$ are calculated by: $\left|\Delta_{h h / v v}\right|=\sigma_{h h} / \sigma_{v v} \quad, \quad\left|\Delta_{h v / v v}\right|=\sigma_{h v} / \sigma_{v v} \quad, \quad$ and $\left|\Delta_{v h / v v}\right|=\sigma_{v h} / \sigma_{v v}$. The co-pol channel imbalance phase is estimated by the phase difference between $\mathrm{HH}$ and VV channels, at peak location after oversampling. However, due to the very low cross-pol trihedral CR return, i.e., $\mathrm{HV}$ and $\mathrm{VH}$, the cross-pol channel imbalance residual cannot be precisely derived from the Inner Mongolia calibration site.

\begin{tabular}{lccccc}
\hline \hline & $\left|\Delta_{h v / v v}\right| \mathrm{dB}$ & $\left|\Delta_{v h / v v}\right| \mathrm{dB}$ & $\left|\Delta_{h h / v v}\right| \mathrm{dB}$ & $\arg \left(\Delta_{h h / v v}\right)$ & $\sigma_{v v} \mathrm{~dB}$ \\
\hline CR. 331-1 & -40.74 & -42.65 & 0.19 & $-1.01^{\circ}$ & 34.39 \\
\hline CR. 331-2 & -39.59 & -39.65 & 0.47 & $0.20^{\circ}$ & 34.74 \\
\hline CR. 331-3 & -39.81 & -36.98 & 0.23 & $-0.01^{\circ}$ & 34.37 \\
\hline CR. 331-4 & -37.07 & -32.99 & -0.21 & $-2.40^{\circ}$ & 34.80 \\
\hline CR. 331-5 & -32.74 & -38.21 & 0.30 & $-2.22^{\circ}$ & 34.71 \\
\hline \hline
\end{tabular}

Table 1. The integral estimation derived crosstalk, channel imbalance and RCS of VV polarization.

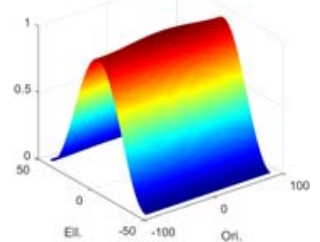

(a)

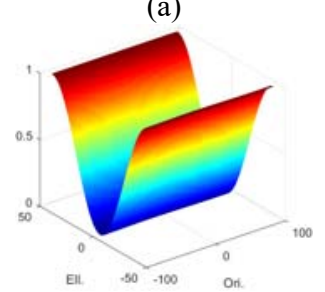

(f)

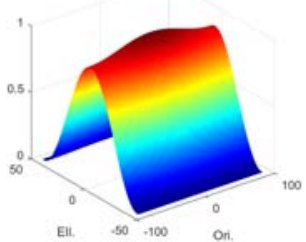

(b)

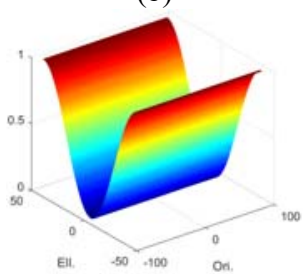

(g)

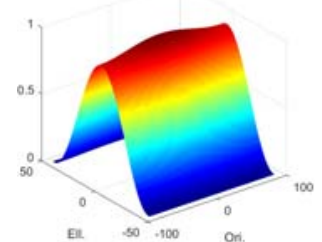

(c)

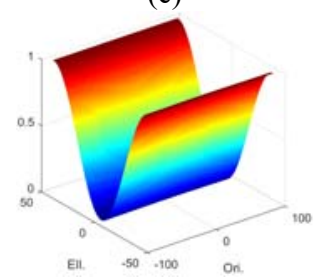

(h)

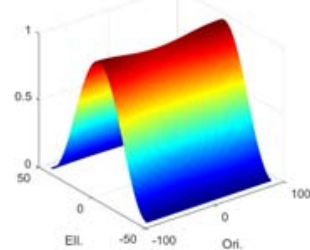

(d)

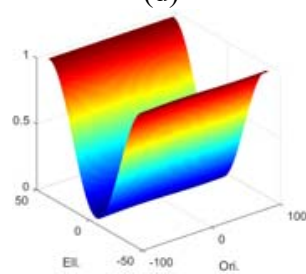

(i)

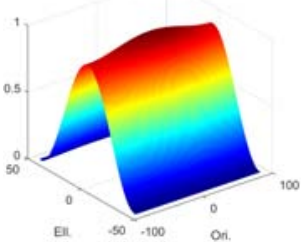

(e)

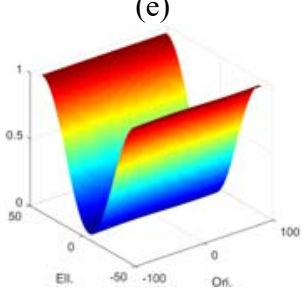

(j)

Figure 2. The polarimetric signature response of 5 trihedral CRs. (a)-(e) represent the co-pol responses from 331-1 to 331-5, and (f)-

(j) represent cross-pol responses. 
After implementing "meta.xml" provided "QualifyValue" and "CalibrationConst" scales, the GeoTiff stored digital values are converted to sigma naught, and we exploit the integration method to estimate the crosstalk, co-pol channel imbalance and RCS of VV polarization in Table. 1. The quantitative estimation shows most crosstalk better than $-35 \mathrm{~dB}$, but CRs of 331-4 and 331-5 sometime present about $-32 \mathrm{~dB}$ crosstalk. The $\mathrm{HH}$ and $\mathrm{VV}$ channel ratio varies from -0.21 to $0.47 \mathrm{~dB}$, which seems better than $0.5 \mathrm{~dB}$ design specification. Because there are a large number of beams designed in GF-3 and the whole calibration process is time cost. Currently, the GF-3 is still monitored in calibration process. The adorable product qualification of beam wave 213 does not mean all data of other beams satisfied the designed specification. The phase difference between $\mathrm{HH}$ and $\mathrm{VV}$ is very low in Table. 1, within 2.5 degree, because the inner calibration circuit is switched on. The GF-3 users are strongly recommended to check whether the circuit is working or not by the label "DoFPInnerImbalanceComp" and "DoFPCalibration" in "meta.xml". When the inner calibration circuit was switched on, the labels are both set as " 1 ". In last column of Table. 1, the RCS values of $5 \mathrm{CRs}$ are estimated at VV channel and we found it very close to $35 \mathrm{~dB}$ theoretical value. The absolute calibration error is smaller than $1 \mathrm{~dB}$. The corresponding co-pol and crosspol polarimetric signature responses of 5 CRs are displayed in Figure. 2. All mentioned performances is presented after 16 times oversampling, because the CR size is much smaller than image resolution and pixel space.

\section{CONCLUSION}

In this paper, we display the GF-3 QPSI I product quality by 5 trihedral CRs in Inner Mongolia. The integration method shows the crosstalk varies from -42.65 to $-32.74 \mathrm{~dB}$, and co-pol channel imbalance varies from -0.21 to $0.47 \mathrm{~dB}$ with phase error -2.34 to 0.20 degree. The CRs solved RCS of VV channel are from 34.37 to $34.80 \mathrm{~dB}$ which is very close to $35 \mathrm{~dB}$ theoretical value. Generally, there are more than hundreds of beans wave in GF-3 satellite and the system calibration work is still going on. Therefore, the product quality of all beams may be not uniform. This paper only evaluate the image of beam code 213 on July 6 , 2017 , but the performance of other beams is still unknown. The comprehensive calibration and evaluation works of most common beams are planned in 2018. When the CRs could be found in scenes, user could consider exploit the nature media to approximately estimate system distortion elements (Van Zyl, 1990; Quegan, 1994; Ainsworth, 2006; Shi, 2014; Fore, 2015).

\section{ACKNOWLEDGEMENTS}

This work was supported by the National Natural Science Foundation under Grants 41501382, 41601355, 41771377 and 61671334 , by the Hubei Provincial Natural Science Foundation of China under Grant 2015CFB328, by the Hong Kong Scholars Program

\section{REFERENCES}

Ainsworth, T. L., Ferro-Famil, L. and Lee, J. S., 2006. Orientation Angle Preserving a Posteriori Polarimetric Sar Calibration. IEEE Transactions on Geoscience and Remote Sensing 44(4), pp. 994-1003.

Fore, A. G., Chapman, B. D., Hawkins, B. P., Hensley, S., Jones, C. E., Michel, T. R. and Muellerschoen, R. J., 2015. Uavsar
Polarimetric Calibration. IEEE Transactions on Geoscience and Remote Sensing 53(6), pp. 3481-3491.

Freeman, A., 1992. Sar Calibration: An Overview. IEEE Transactions on Geoscience and Remote Sensing 30(6), pp. 1107-1121.

Gray, A. L., Vachon, P. W., Livingstone, C. E. and Lukowski, T. I., 1990. Synthetic Aperture Radar Calibration Using Reference Reflectors. IEEE Transactions on Geoscience and Remote Sensing 28(3), pp. 374-383.

Hu, J., Ding, X. L., Zhang, L., Sun, Q., Li, Z. W., Zhu, J. J. and Lu, Z., 2017. Estimation of 3-D Surface Displacement Based on Insar and Deformation Modeling. IEEE Transactions on Geoscience and Remote Sensing 55(4), pp. 2007-2016.

Quegan, S., 1994. A Unified Algorithm for Phase and Cross-Talk Calibration of Polarimetric Data-Theory and Observations. IEEE Transactions on Geoscience and Remote Sensing 32(1), pp. 8999.

Shi, L., Sun, W., Yang, J., Li, P. and Lu, L., 2015. Building Collapse Assessment by the Use of Postearthquake Chinese Vhr Airborne Sar. IEEE Geoscience and Remote Sensing Letters 12(10), pp. 2021-2025.

Shi, L., Yang, J. and Li, P., 2014. Co-Polarization Channel Imbalance Determination by the Use of Bare Soil. ISPRS Journal of Photogrammetry and Remote Sensing 95(1), pp. 53-67.

Shi, L., Zhang, L., Zhao, L., Li, P. and Wu, D., 2016. Adaptive Laplacian Eigenmap-Based Dimension Reduction for Ocean Target Discrimination. IEEE Geoscience and Remote Sensing Letters 13(7), pp. 902-906.

Sun, W., Shi, L., Yang, J. and Li, P., 2016. Building Collapse Assessment in Urban Areas Using Texture Information from Postevent Sar Data. IEEE Journal of Selected Topics in Applied Earth Observations and Remote Sensing 9(8), pp. 3792-3808.

Van Zyl, J. J., 1990. Calibration of Polarimetric Radar Images Using Only Image Parameters and Trihedral Corner Reflector Responses. IEEE Transactions on Geoscience and Remote Sensing 28(3), pp. 337-348.

Voronovich, A. G. and Zavorotny, V. U., 2017. Measurement of Ocean Wave Directional Spectra Using Airborne Hf/Vhf Synthetic Aperture Radar: A Theoretical Evaluation. IEEE Transactions on Geoscience and Remote Sensing 55(6), pp. 3169-3176.

Zhang, Q., 2017. System Design and Key Technologies of the Gf-3 Satellite. Acta Geodaetica et Cartographica Sinica 46(03), pp. 269-277. 